\title{
Solvable Lattice Models Related to the Vector Representation of Classical Simple Lie Algebras
}

\author{
Michio Jimbo, Tetsuji Miwa, and Masato Okado \\ Research Institute for Mathematical Sciences, Kyoto University, Kyoto 606, Japan
}

\begin{abstract}
A series of solvable lattice models with face interaction are introduced on the basis of the affine Lie algebra $X_{n}^{(1)}=A_{n}^{(1)}, B_{n}^{(1)}, C_{n}^{(1)}, D_{n}^{(1)}$. The local states taken on by the fluctuation variables are the dominant integral weights of $X_{n}^{(1)}$ of a fixed level. Adjacent local states are subject to a condition related to the vector representation of $X_{n}$. The Boltzmann weights are parametrized by elliptic theta functions and solve the star-triangle relation.
\end{abstract}

\section{Introduction}

Through the last decade of investigation the significance of the Yang-Baxter equation (YBE) in integrable systems has been commonly acknowledged. A number of two dimensional models have been solved in statistical mechanics and in quantum field theory on this basis. Very recently there is also renewed interest for the YBE because of its connection with other branches of mathematics, such as the braid group, link invariants and operator algebras [1-4].

In the study of the YBE, the idea of Lie algebras and representation theory has turned out to be particularly fruitful. Such a viewpoint has been developed in the framework of the quantum inverse method. Motivated by the connection with soliton theory, a quasi-classical version of the YBE was formulated (the classical YBE). The classification of its solutions associated with simple Lie algebras was accomplished by Belavin and Drinfeld [5]. Kulish et al. [6] initiated the representation theoretical construction of quantum $R$ matrices ( $=$ solutions to the YBE) corresponding to the classical ones. These works led several authors [7-11] to the discovery of a novel algebraic structure underlying the problem, the quantum group as formulated by Drinfeld [9].

In the statistical mechanics language, the works mentioned above are concerned with the vertex model. Here the fluctuation variables are placed on the edges of a two dimensional lattice, and each element of the $R$ matrix provides the statistical weight (the Boltzmann weight) for a configuration round a lattice site, or a vertex. There is also a dual object, the face model, in which the variables live on the sites, and the Boltzmann weight is attached to a configuration round a face. 
(This was introduced by Baxter [12] and was called the interaction-round-a-face model. Here we prefer a simpler terminology, the face model.) The YBE is formulated accordingly and is more often called the star-triangle relation (STR).

Baxter's solution of the hard hexagon model [13] showed that the face model has interest of its own right. For face models one can apply Baxter's corner transfer matrix method to evaluate the local state probability (LSP), the probability that the variable at a site takes a particular state. Andrews et al. [14] presented a series of face models generalizing the hard hexagon model, and obtained their LSPs in terms of modular functions. Subsequently it was recognized [15] that their models are naturally associated with the affine Lie algebra $A_{1}^{(1)}$, and that the modular functions in the LSP result are the branching coefficients for the pair $\left(A_{1}^{(1)} \oplus A_{1}^{(1)}\right.$, $\left.A_{1}^{(1)}\right)$. This sort of result was first obtained in the $\mathbb{Z}_{n}$ Ising type models [16], and has been further extended to other cases; those related to symmetric tensors of $A_{1}^{(1)}$ $[17,18]$ or to the vector representation of $A_{n}^{(1)}[19,20]$ (in the sense to be described below).

This paper is directly motivated by these latest works. Before giving a detailed introduction of the theme let us mention our motivation in relation to the conformal field theory. The enumeration of all the solvable conformal field theories is a goal still far from our sight. Recently, the classification of the unitary discrete series has been completed, in which the conformal anomaly satisfies $0<c<1$. Aiming at theories with $c \geqq 1$ three different approaches are being pursued. The first is to exploit larger symmetries than the Virasoro algebra such as affine Lie algebras, super Virasoro algebras, parafermion algebras, $W_{n}$ algebras and so on. The second is to approach from the modular invariance. The third is to work with solvable lattice models. One advantage in the third approach is that the master equation is at hand; the YBE or the STR. Recent studies [21-30] have revealed that the known solutions are just the tip of the iceberg. The aim of this paper is to manifest its body by showing the role of affine Lie algebras in this game.

Consider an affine Lie algebra of type $X_{n}^{(1)}\left(X_{n}=A_{n}, B_{n}, C_{n}\right.$ or $\left.D_{n}\right)$ and an irreducible representation $\left(\pi, V_{\pi}\right)$ of its classical part $X_{n}$. We put forward the construction of solvable face models that have the following features: Firstly, the local states, i.e. the values of the fluctuation variables, are taken to be the dominant integral weights of $X_{n}^{(1)}$ of a fixed level $l$. We choose $l$ to be not too small so that there exists the level $l$ highest weight representation of $X_{n}^{(1)}$ whose highest weight vector generates an irreducible $X_{n}$ module isomorphic to $V_{\pi}$.

Secondly, we require that the states $(a, b)$ of neighboring variables should be admissible in the following sense. Let $V(\bar{b})$ denote the irreducible $X_{n}$ module having the classical part $\bar{b}$ of $b$ as the highest weight. We say that

$(a, b)$ is admissible if and only if $V(\overline{\sigma(b)})$ appears in the irreducible decomposition of $V(\overline{\sigma(a)}) \otimes V_{\pi}$ for any Dynkin diagram automorphism $\sigma$.

The condition $(*)$ was introduced in $[25,26]$ to rule out the divergent restricted weights, and was shown to fit the description of the LSP result in terms of the affine Lie algebra characters $[17,18,20]$. It was also utilized by Wenzl [31] in his construction of the irreducible representations of the Hecke algebra, and by Tsuchiya and Kanie [32] in the fusion rule of vertex operators in the conformal 
field theory. Recently, Pasquier [33] touched upon the possibility of constructing face models of this sort.

In general, the solution to the star-triangle relation satisfying these two requirements is not unique (see Sect. 5). Therefore we need a further specification of the model. In the $A_{n}^{(1)}$ family [19] it was noticed that the factors $G_{a}$ appearing in the second inversion relation [see $(2.13 \mathrm{~b})]$ are equal to the principally specialized characters. We exploit this remarkable coincidence as the third Merkmal in finding solutions of type $X_{n}^{(1)}$

The cases $X_{n}^{(1)}=A_{n}^{(1)}$ and $\pi=$ the vector representation $[19,20]$ or symmetric tensors $[26,27]$ have been treated previously. Here we shall work out the solutions in the case $X_{n}^{(1)}=B_{n}^{(1)}, C_{n}^{(1)}, D_{n}^{(1)}$ and $\pi=$ the vector representation. In fact, as was done by Andrews et al. [14] in the original 8VSOS model, we shall first construct models such that the local states take generic complex weights in the dual space of the Cartan subalgebra. The admissibility $(*)$ is replaced by a weaker condition that $b-a$ is a weight appearing in $V_{\pi}$. The solutions of the STR are parametrized by degree 2 elliptic theta functions, in contrast to the degree 1 parametrization in the $A_{n}^{(1)}$ family. The resulting models (which we call unrestricted models) admit infinitely many local states, differing mutually by integral weights. We then restrict them to dominant integral weights of level $l$ and show that the STR closes among the finitely many Boltzmann weights thus obtained. These are the main results of the present article.

Let us include one remark on the above formulation of the face model. The representations $\left(\pi, V_{\pi}\right)$ of $X_{n}$ dealt with in this paper are limited in that the weight multiplicities are merely one. For general $\pi$ we need presumably to introduce extra fluctuation variables on edges corresponding to the weight multiplicities.

The text is organized as follows. In Sect. 2 we give the formulas for the Boltzmann weights for the unrestricted models of type $A_{n}^{(1)}, B_{n}^{(1)}, C_{n}^{(1)}$, and $D_{n}^{(1)}$. Proof of the STR is given in Sect. 3. Section 4 deals with the restriction process described above. Discussions are included in Sect. 5.

\section{Unrestricted Models}

The models we consider will be built upon the affine Lie algebra $X_{n}^{(1)}$, where $X_{n}$ denotes one of the finite dimensional simple Lie algebras $A_{n}, B_{n}, C_{n}$, or $D_{n}$. Our basic reference on the affine Lie algebras is Kac-Peterson [34]. We denote by $\Lambda_{j}$ $(0 \leqq j \leqq n)$ the fundamental weights, and set $\varrho=\Lambda_{0}+\ldots+\Lambda_{n}, \mathfrak{h}_{\mathbb{C}}^{*}=\sum_{j=0}^{n} \mathbb{C} \Lambda_{j}$. For an element $a \in \mathfrak{h}_{\mathbb{C}}^{*}, \bar{a}$ signifies its calssical part. Following Bourbaki [35] we introduce orthonormal vectors $\varepsilon_{i}$ to express $\bar{\Lambda}_{j}$ as in Table 1 . We list also the set $\mathscr{A}$ of weights that belong to the vector representation of $X_{n}$.

We write an element of $\mathscr{A}$ as

$$
\begin{aligned}
\hat{\mu} & =\varepsilon_{\mu}-\frac{1}{n+1}\left(\varepsilon_{1}+\ldots+\varepsilon_{n+1}\right) \quad(1 \leqq \mu \leqq n+1) \text { for } A_{n}, \\
& = \pm \varepsilon_{i} \text { or } 0 \quad(\mu= \pm i, 1 \leqq i \leqq n, \text { or } \mu=0) \text { for } B_{n}, \\
& = \pm \varepsilon_{i} \quad(\mu= \pm i, 1 \leqq i \leqq n) \text { for } C_{n}, D_{n} .
\end{aligned}
$$


Table 1

\begin{aligned} \hline$A_{n} \quad \mathscr{A} & =\left\{\varepsilon_{1}-\varepsilon, \ldots, \varepsilon_{n+1}-\varepsilon\right\} \\ , \bar{\Lambda}_{i} & =\varepsilon_{1}+\ldots+\varepsilon_{i}-i \varepsilon \quad(1 \leqq i \leqq n), \quad \varepsilon=\frac{1}{n+1} \sum_{j=1}^{n+1} \varepsilon_{j} \\ . \mathscr{A} & =\left\{ \pm \varepsilon_{1}, \ldots, \pm \varepsilon_{n}, 0\right\} \\ , B_{n} \quad(1 \leqq i \leqq n-1) & , \bar{A}_{i}=\varepsilon_{1}+\ldots+\varepsilon_{i} \quad(1=n) \\ & .=\frac{1}{2}\left(\varepsilon_{1}+\ldots+\varepsilon_{n}\right) \quad(i=1 \leqq \\ C_{n} \quad & \mathscr{A}=\left\{ \pm \varepsilon_{1}, \ldots, \pm \varepsilon_{n}\right\} \\ , \bar{A}_{i} & =\varepsilon_{1}+\ldots+\varepsilon_{i} \quad(1 \leqq i \leqq n) \\ . \mathscr{A} & =\left\{ \pm \varepsilon_{1}, \ldots, \pm \varepsilon_{n}\right\} \\ , \bar{A}_{i} & =\varepsilon_{1}+\ldots+\varepsilon_{i} \quad(1 \leqq i \leqq n-2) \\ & ,=\frac{1}{2}\left(\varepsilon_{1}+\ldots+\varepsilon_{n-2}+\varepsilon_{n-1}-\varepsilon_{n}\right) \quad(i=n-1) \\ & ,=\frac{1}{2}\left(\varepsilon_{1}+\ldots+\varepsilon_{n-2}+\varepsilon_{n-1}+\varepsilon_{n}\right) \quad(i=n)\end{aligned}$.

For $a \in \mathfrak{h}_{\mathbb{C}}^{*}$ we put

$$
\begin{aligned}
a_{\mu} & =\langle a+\varrho, \hat{\mu}\rangle \quad(\mu \neq 0), \\
& =-\frac{1}{2} \quad(\mu=0),
\end{aligned}
$$

so that we have

$$
\begin{aligned}
\bar{a}+\bar{\varrho} & =\sum_{i=1}^{n+1} a_{i} \varepsilon_{i}, \sum_{i=1}^{n+1} a_{i}=0 \text { for } A_{n}^{(1)}, \\
& =\sum_{i=1}^{n} a_{i} \varepsilon_{i} \text { for } B_{n}^{(1)}, C_{n}^{(1)}, D_{n}^{(1)} .
\end{aligned}
$$

Consider now a two dimensional square lattice $\mathscr{L}$. We shall introduce face models on $\mathscr{L}$ that have the following basic features:

(1) The fluctuation variable placed on each lattice site assumes its values in $\mathfrak{b}_{\mathbb{C}}^{*}$. We call these values local states.

(2) Adjacent local states differ by an element in $\mathscr{A}$, i.e. by a weight in the vector representation of $X_{n}$. More precisely, this means that the Boltzmann weights $W\left(\begin{array}{ll}a & b \\ d & c\end{array}\right)$ describing the interaction of four fluctuation variables round a face [12] satisfy the condition

$$
W\left(\begin{array}{ll}
a & b \\
d & c
\end{array}\right)=0 \text { unless } \quad b-a, c-b, d-a, c-d \in \mathscr{A} .
$$

Because of (2.1) the local states appearing in a possible configuration on $\mathscr{L}$ are actually confined to the set $a^{0}+\sum_{\hat{\mu} \in \mathscr{A}} \mathbb{Z} \hat{\mu}$, where $a^{0}$ is a fixed element of $\mathfrak{h}_{\mathbb{C}}^{*}$.

Under the setting above we have found a system of Boltzmann weights $W\left(\begin{array}{ll}a & b \\ d & c\end{array} \mid u\right)$ that depend on the spectral parameter $u \in \mathbb{C}$ and solve the star-triangle 
relation $(\mathrm{STR})$

$$
\begin{aligned}
& \sum_{g} W\left(\begin{array}{ll}
f & g \\
e & d
\end{array} \mid u\right) W\left(\begin{array}{ll}
b & c \\
g & d
\end{array} \mid v\right) W\left(\begin{array}{ll}
a & b \\
f & g
\end{array} \mid u+v\right) \\
& =\sum_{g} W\left(\begin{array}{ll}
a & b \\
g & c
\end{array} \mid u\right) W\left(\begin{array}{ll}
a & g \\
f & e
\end{array} \mid v\right) W\left(\begin{array}{ll}
g & c \\
e & d
\end{array} \mid u+v\right) .
\end{aligned}
$$

The solutions are parametrized in terms of the elliptic theta function

$$
\begin{gathered}
{[u]=\theta_{1}\left(\frac{\pi u}{L}, p\right),} \\
\theta_{1}(u, p)=2 p^{1 / 8} \sin u \prod_{k=1}^{\infty}\left(1-2 p^{k} \cos 2 u+p^{2 k}\right)\left(1-p^{k}\right),
\end{gathered}
$$

where $L \neq 0$ is an arbitrary complex parameter. Explicitly they are given by the following formulas. We shall write

$$
\mu \square_{v}^{\kappa} \sigma=\mathrm{W}\left(\begin{array}{cc}
a & a+\hat{\kappa} \\
a+\hat{\mu} & a+\hat{\mu}+\hat{v}
\end{array} \mid u\right) \quad(\hat{\mu}+\hat{v}=\hat{\kappa}+\hat{\sigma}) .
$$

$A_{n}^{(1)}$ :

$$
\begin{gathered}
\mu \square_{\mu}^{\mu} \mu=\frac{[1+u]}{[1]}, \\
\mu \square_{v}^{\mu} v=\frac{\left[a_{\mu}-a_{v}-u\right]}{\left[a_{\mu}-a_{v}\right]} \quad(\mu \neq v), \\
\mu \underset{v}{\square} \mu=\frac{[u]}{[1]}\left(\frac{\left[a_{\mu}-a_{v}+1\right]\left[a_{\mu}-a_{v}-1\right]}{\left[a_{\mu}-a_{v}\right]^{2}}\right)^{1 / 2} \quad(\mu \neq v) .
\end{gathered}
$$

$B_{n}^{(1)}, C_{n}^{(1)}, D_{n}^{(1)}$ :

$$
\begin{aligned}
& \mu \square_{\mu}^{\mu} \mu=\frac{[\lambda-u][1+u]}{[\lambda][1]} \quad(\mu \neq 0) \\
& \mu \square_{v}^{\mu} v=\frac{[\lambda-u]\left[a_{\mu}-a_{v}-u\right]}{[\lambda]\left[a_{\mu}-a_{v}\right]} \quad(\mu \neq \pm v) \\
& \mu \square_{\nu}^{\nu} \mu=\frac{[\lambda-u][u]}{[\lambda][1]}\left(\frac{\left[a_{\mu}-a_{\nu}+1\right]\left[a_{\mu}-a_{v}-1\right]}{\left[a_{\mu}-a_{\nu}\right]^{2}}\right)^{1 / 2} \quad(\mu \neq \pm v), \\
& \mu \square_{-\mu}^{v}-v=\frac{[u]\left[a_{\mu}+a_{v}+1+\lambda-u\right]}{[\lambda]\left[a_{\mu}+a_{v}+1\right]}\left(G_{a \mu} G_{a v}\right)^{1 / 2} \quad(\mu \neq v),
\end{aligned}
$$




$$
\begin{gathered}
\mu \square_{-\mu}^{\mu}-\mu=\frac{[\lambda+u]\left[2 a_{\mu}+1+2 \lambda-u\right]}{[\lambda]\left[2 a_{\mu}+1+2 \lambda\right]}-\frac{[u]\left[2 a_{\mu}+1+\lambda-u\right]}{[\lambda]\left[2 a_{\mu}+1+2 \lambda\right]} H_{a \mu}, \\
=\frac{[\lambda-u]\left[2 a_{\mu}+1-u\right]}{[\lambda]\left[2 a_{\mu}+1\right]}+\frac{[u]\left[2 a_{\mu}+1+\lambda-u\right]}{[\lambda]\left[2 a_{\mu}+1\right]} G_{a \mu}(\mu \neq 0), \\
H_{a \mu}=\sum_{\kappa(\neq \mu)} \frac{\left[a_{\mu}+a_{\kappa}+1+2 \lambda\right]}{\left[a_{\mu}+a_{\kappa}+1\right]} G_{a \kappa} .
\end{gathered}
$$

Here $\mu, v=1, \ldots, n+1$ (for $\left.A_{n}^{(1)}\right), 0, \pm 1, \ldots, \pm n$ (for $B_{n}^{(1)}$ ) or $\pm 1, \ldots, \pm n$ (for $C_{n}^{(1)}$, $\left.D_{n}^{(1)}\right)$. In the above we used the following notations. The crossing parameter $\lambda$ in (2.5) is fixed to be

$$
\lambda=-\operatorname{tg} / 2
$$

with $t=(\text { long root })^{2} / 2$ and $g=$ the dual Coxeter number of $X_{n}^{(1)}$ (see Table 2). The factor $G_{a \mu}$ is given by

$$
\begin{aligned}
G_{a \mu} & =G_{a+\hat{\mu}} / G_{a}=\sigma \frac{h\left(a_{\mu}+1\right)}{h\left(a_{\mu}\right)} \prod_{\kappa(\neq \pm \mu, 0)} \frac{\left[a_{\mu}-a_{\kappa}+1\right]}{\left[a_{\mu}-a_{\kappa}\right]}(\mu \neq 0), \quad G_{a 0}=1, \\
G_{a} & =\prod_{1 \leqq i<j \leqq n+1}\left[a_{t}-a_{j}\right] \text { for } A_{n}^{(1)}, \\
& =\varepsilon(a) \prod_{i=1}^{n} h\left(a_{i}\right) \prod_{1 \leqq i<j \leqq n}\left[a_{i}-a_{j}\right]\left[a_{i}+a_{j}\right] \text { for } B_{n}^{(1)}, C_{n}^{(1)}, D_{n}^{(1)} .
\end{aligned}
$$

Here $\sigma=-1$ for $C_{n}^{(1)}$ and $=1$ otherwise, and $\varepsilon(a)$ is a sign factor such that $\varepsilon(a+\hat{\mu}) / \varepsilon(a)=\sigma$. The function $h(a)$ is given in Table 2. Up to a common factor independent of $a$, the expression for $\pm G_{a}$ coincides with the denominator formula of $X_{n}^{(1)}$ evaluated at $a+\varrho$, and hence with the principally specialized character for the dual affine Lie algebra (see [34] for the definition of the dual affine Lie algebra).

Table 2

\begin{tabular}{lllll}
\hline type & $A_{n}^{(1)}$ & $B_{n}^{(1)}$ & $C_{n}^{(1)}$ & $D_{n}^{(1)}$ \\
$g$ & $n+1$ & $2 n-1$ & $n+1$ & $2 n-2$ \\
$t$ & 1 & 1 & 2 & 1 \\
$h(a)$ & 1 & {$[a]$} & {$[2 a]$} & 1 \\
\hline
\end{tabular}

Proof of the STR (2.2) [including that of the equality of (2.5e) and (2.5f) for $\mu \neq 0]$ will be given in Sect. 3. Besides the spectral parameter $u$, these weights contain two arbitrary parameters $L, p$ entering in (2.3). We call the models defined by $(2.1,4-5)$ unrestricted $X_{n}^{(1)}$ models (as opposed to restricted models to be discussed in Sect. 4). These terminologies, unrestricted and restricted, go back to Andrews-Baxter-Forrester [14].

We have verified that the following equivalences between the representations of classical simple Lie algebras extend to those between the corresponding unrestricted face models: 


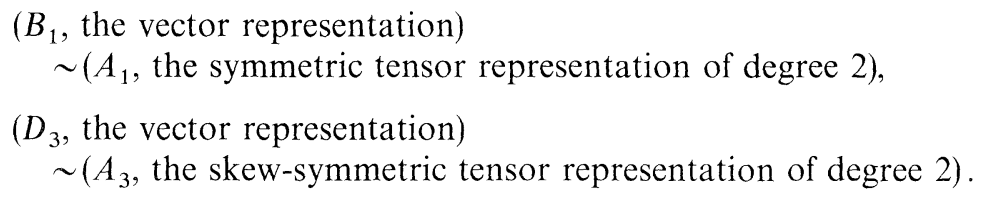

The symmetric tensor representations of the $A_{n}^{(1)}$ face model have been constructed in $[26,27]$. We hope to discuss a similar construction for the skew-symmetric tensors in a future publication (see [36] for the vertex models).

The Boltzmann weights enjoy the following properties.

Initial condition

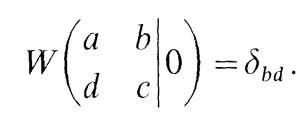

Reflection symmetry

$$
W\left(\begin{array}{ll}
a & b \\
d & c
\end{array} \mid u\right)=W\left(\begin{array}{ll}
a & d \\
b & c
\end{array} \mid u\right) .
$$

Dynkin diagram symmetry

$$
W\left(\begin{array}{ll}
\sigma(a) & \sigma(b) \\
\sigma(d) & \sigma(c)
\end{array} \mid u\right)=W\left(\begin{array}{ll}
a & b \\
d & c
\end{array} \mid u\right)
$$

where $\sigma$ is any Dynkin diagram automorphism acting on $\mathfrak{h}_{\mathbb{C}}^{*}$ and the parameter $L$ is set to $t(l+g)(l$ : the level of $a)$.

Rotational symmetry (valid except for $A_{n}^{(1)}$ )

$$
W\left(\begin{array}{ll}
a & b \\
d & c
\end{array} \mid u\right)=\left(\frac{G_{b} G_{d}}{G_{a} G_{c}}\right)^{1 / 2} W\left(\begin{array}{ll}
d & a \\
c & b
\end{array} \mid \lambda-u\right) .
$$

The following inversion relations will play a role in the evaluation of the local state probabilities, though we do not discuss it in this paper.

$$
\begin{gathered}
\sum_{g} W\left(\begin{array}{ll}
a & g \\
d & c
\end{array} \mid u\right) W\left(\begin{array}{ll}
a & b \\
g & c
\end{array} \mid-u\right)=\delta_{b d} \varrho_{1}(u), \\
\sum_{g} \bar{W}\left(\begin{array}{ll}
a & b \\
d & g
\end{array} \mid \lambda-u\right) \bar{W}\left(\begin{array}{ll}
c & d \\
b & g
\end{array} \mid \lambda+u\right)=\delta_{a c} \varrho_{2}(u) .
\end{gathered}
$$

Here we have set

$$
\begin{gathered}
\bar{W}\left(\begin{array}{ll}
a & b \\
d & c
\end{array} \mid u\right)=\left(\frac{G_{a} G_{c}}{G_{b} G_{d}}\right)^{1 / 2} W\left(\begin{array}{ll}
a & b \\
d & c
\end{array} \mid u\right), \\
\varrho_{1}(u)=\frac{[1+u][1-u]}{[1]^{2}}, \quad \varrho_{2}(u)=\frac{[\lambda+u][\lambda-u]}{[1]^{2}} \text { for } A_{n}^{(1)}, \\
\varrho_{1}(u)=\varrho_{2}(u)=\frac{[\lambda+u][\lambda-u][1+u][1-u] \text { for } B_{n}^{(1)}, C_{n}^{(1)}, D_{n}^{(1)} .}{[\lambda]^{2}[1]^{2}}
\end{gathered}
$$


In fact the first inversion relation (2.13a) is a direct consequence of the STR (2.2) and the initial condition (2.9). For types other than $A_{n}^{(1)}$ (see [20] for $A_{n}^{(1)}$ ), the second inversion relation (2.13b) follows from (2.12) and (2.13a).

\section{Proof of the STR}

This section is devoted to the proof of the STR (2.2). Since a proof in the case of $A_{n}^{(1)}$ was given in a more general situation [27], we only consider the remaining cases $B_{n}^{(1)}, C_{n}^{(1)}$, and $D_{n}^{(1)}$. Throughout this section $L, \tau$ are complex numbers satisfying $L \neq 0, \operatorname{Im} \tau>0\left[p=e^{2 \pi i \tau}\right.$ in $\left.(2.3)\right]$. We shall frequently use the quasi-periodicity property of the symbol $[u]$

$$
[u+L]=-[u], \quad[u+L \tau]=-e^{-\pi i \tau-2 \pi i u / L}[u],
$$

along with the following standard lemma (cf. [37]).

Lemma 1. If $f(u)$ is entire, not identically zero and satisfies

$$
f(u+L)=e^{-2 \pi i B} f(u), \quad f(u+L \tau)=e^{-2 \pi i\left(A_{1}+A_{2} u / L\right)} f(u),
$$

then $A_{2}$ is a non-negative integer, $f(u)$ has $A_{2}$ zeros $\bmod L \mathbb{Z}+L \mathbb{Z} \tau$ and

$\sum$ zeros $\equiv L\left(B \tau+A_{2} / 2-A_{1}\right)$.

First we prove the inversion relation (2.13a). It is divided into the following three types:

\section{Proposition 2.}

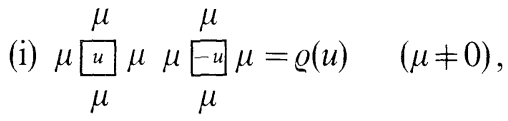

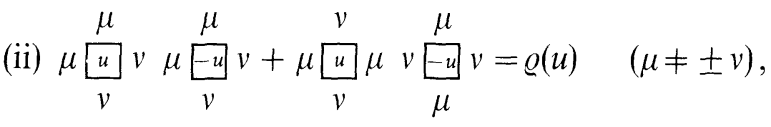

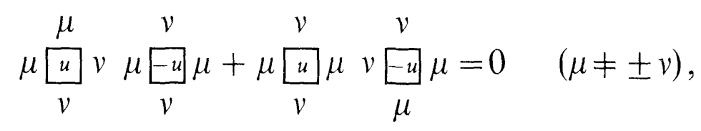

(iii) $\sum_{k} \frac{\kappa}{\mu-\mu}-\kappa \kappa \underset{-\kappa}{v-u}-v=\varrho(u) \delta_{\mu \nu}$,

where

$$
\varrho(u)=\frac{[\lambda+u][\lambda-u][1+u][1-u]}{[\lambda]^{2}[1]^{2}} .
$$

In (iii) the sum is taken over $\kappa= \pm 1, \ldots, \pm n, 0$ for $B_{n}^{(1)}$, and $= \pm 1, \ldots, \pm n$ for $C_{n}^{(1)}$, $D_{n}^{(1)}$.

Before going to the proof we need to prepare two lemmas. 
Lemma 3. For any $a, b, c, u, v, w, \lambda(u+v+w=\lambda)$, we have the identity

$$
\begin{aligned}
0= & -[\lambda-u][\lambda-v][\lambda-w] \frac{[b+c+u][c+a+v][a+b+w]}{[b+c][c+a][a+b]} \\
& +[\lambda-u][v][w] \frac{[2 a-u][a+b+\lambda-v][a+c+\lambda-w]}{[2 a][a+b][a+c]} \\
& +[u][\lambda-v][w] \frac{[b+a+\lambda-u][2 b-v][b+c+\lambda-w]}{[b+a][2 b][b+c]} \\
& +[u][v][\lambda-w] \frac{[c+a+\lambda-u][c+b+\lambda-v][2 c-w]}{[c+a][c+b][2 c]} \\
& +[u][v][w] \sum_{\omega} \frac{1}{2} \frac{[a+\lambda-u+\omega][b+\lambda-v+\omega][c+\lambda-w+\omega]}{[a+\omega][b+\omega][c+\omega]} e^{2 \pi i \theta(\omega)} .
\end{aligned}
$$

Here the summation $\sum_{\omega}$ is over the half periods $\omega=0, L / 2, L \tau / 2, L(1+\tau) / 2$, and

$$
\begin{aligned}
\theta(\omega) & =0 & & \omega=0, L / 2, \\
& =\lambda / L & & \omega=L \tau / 2, L(1+\tau) / 2 .
\end{aligned}
$$

Proof. Regarding the right-hand side as a function of $a$, one can verify that it does not have any pole. Next we apply Lemma 1 , taking $B=A_{2}=0, A_{1}=(v+w) / L$.

\section{Lemma 4. Set}

$$
\varphi(u)=\frac{1}{C} \frac{d}{d u} \log [u], \quad C=\lim _{u \rightarrow 0} \frac{[u]}{u} .
$$

We have then

$$
\varphi(u+1)+\varphi(u-1)-2 \varphi(u)=\frac{[1]^{2}[2 u]}{[u]^{2}[u-1][u+1]} .
$$

Proof. By the definition, the function $\varphi(u)$ satisfies

$$
\varphi(u+L)=\varphi(u), \quad \varphi(u+L \tau)=\varphi(u)-2 \pi i / L C .
$$

From this one can verify that (the left-hand side)-(the right-hand side) of (3.4) is a doubly periodic odd function and has no poles. Hence it must be zero.

Proof of Proposition 2. Equation (i) is trivial. Equation (ii) is easily checked by using the addition formula,

$$
\begin{aligned}
& {[x+z][x-z][y+w][y-w]-[x+w][x-w][y+z][y-z]} \\
& \quad=[x+y][x-y][z+w][z-w] .
\end{aligned}
$$

Let us prove (iii) with $\mu, v \neq 0$. In this case we use the expression (2.5f). Set

$$
F_{\mu \nu}(z)=\frac{\left[a_{\mu}+z+1+\lambda-u\right]\left[a_{v}+z+1+\lambda+u\right]}{\left[a_{\mu}+z+1\right]\left[a_{v}+z+1\right]} \frac{\sigma h(z+1)[2 z]}{[1] h(z)[2 z+1]} \prod_{\kappa(\neq 0)} \frac{\left[z-a_{\kappa}+1\right]}{\left[z-a_{\kappa}\right]} .
$$

From (2.6) and Table 2, one finds that $F_{\mu v}(z)$ is a doubly periodic function. If $\mu \neq v$, its poles are located at $z=a_{\kappa}(\kappa \neq 0),-1 / 2+\omega(\omega=0, L / 2, L \tau / 2, L(1+\tau) / 2)$. If $\mu=v$, 
it has an additional pole at $z=-a_{\mu}-1$. The relation $\sum \operatorname{Res} F_{\mu \nu}(z)=0$ gives rise to

$$
\begin{aligned}
\sum_{k} & \frac{\left[a_{\mu}+a_{\kappa}+1+\lambda-u\right]\left[a_{v}+a_{\kappa}+1+\lambda+u\right]}{\left[a_{\mu}+a_{\kappa}+1\right]\left[a_{v}+a_{\kappa}+1\right]} G_{a \kappa} \\
- & -\frac{1}{2} \sum_{\omega} \frac{\left[a_{\mu}+1 / 2+\lambda-u+\omega\right]\left[a_{v}+1 / 2+\lambda+u+\omega\right]}{\left[a_{\mu}+1 / 2+\omega\right]\left[a_{v}+1 / 2+\omega\right]} e^{2 \pi i \theta(\omega)} \\
& -\delta_{\mu \nu} \frac{[\lambda-u][\lambda+u]\left[2 a_{\mu}\right]\left[2 a_{\mu}+2\right]}{[1]^{2}\left[2 a_{\mu}+1\right]^{2}} G_{a \mu}^{-1}=0
\end{aligned}
$$

Here $\theta(\omega)$ is given in (3.2), and the summation $\sum_{\omega}$ is over $\omega=0, L / 2, L \tau / 2, L(1+\tau) / 2$. (Note again that the sum $\sum_{\kappa}$ includes $\kappa=0$ for $B_{n}^{(1)}$.) Combining (3.5) with Lemma 3 specialized as $a=b=a_{\mu}+1 / 2, u=-v, w=\lambda$, we obtain (iii) with $\mu, v \neq 0$.

Next let us prove (iii) in the case of $\mu=v=0$. Let $f(u)$ be (the left-hand side)-(the right-hand side) of (iii). In view of Lemma 1, it suffices to check $f(0)$ $=f( \pm \lambda)=f( \pm 1)=0$. (Note that $f(u)$ is an even function.) The only nontrivial step is to show $f(1)=0$. Pick any index $\mu \neq 0$. Regarding $f(1)$ as a function of $a_{\mu}$ we denote it by $g\left(a_{\mu}\right)$, namely

$$
\begin{aligned}
g\left(a_{\mu}\right)= & \left(\frac{[\lambda+1][2 \lambda-1]}{[\lambda][2 \lambda]}-\frac{[1][\lambda-1]}{[\lambda][2 \lambda]} H_{a 0}\right) \\
& \times\left(\frac{[\lambda-1][2 \lambda+1]}{[\lambda][2 \lambda]}+\frac{[1][\lambda+1]}{[\lambda][2 \lambda]} H_{a 0}\right) \\
& -\frac{[1]^{2}}{[\lambda]^{2}} \sum_{\kappa(\neq 0)} \frac{\left[a_{\kappa}+3 / 2+\lambda\right]\left[a_{\kappa}-1 / 2+\lambda\right]}{\left[a_{\kappa}+1 / 2\right]^{2}} G_{a \kappa} .
\end{aligned}
$$

This is doubly periodic in $a_{\mu}$. Let us show that it is holomorphic everywhere. It is easy to see that the apparent poles $a_{\mu}=0$ or $a_{\mu}=a_{\kappa}(\mu \neq \pm \kappa)$ are regular points, and that the coefficient of $\left(a_{\mu}+1 / 2\right)^{-2}$ at $a_{\mu}=-1 / 2$ vanishes. The vanishing of the coefficient of $\left(a_{\mu}+1 / 2\right)^{-1}$ means

$$
\begin{aligned}
0= & -\frac{[1][\lambda+1]^{2}[2 \lambda-1]}{[\lambda]^{2}[2 \lambda]}+\frac{[1][\lambda-1]^{2}[2 \lambda+1]}{[\lambda]^{2}[2 \lambda]}-\frac{[1]^{2}[\lambda-1][\lambda+1]}{[\lambda]^{2}} \\
& \times\left\{2 \varphi(2 \lambda)-\varphi(\lambda-1)-\varphi(\lambda+1)+2 \varphi(1 / 2)+2 \sum_{\kappa(\neq \pm \mu, 0)} \varphi\left(1 / 2-a_{\kappa}\right)\right\} \\
& +\frac{2[1]^{2}[\lambda-1][\lambda+1]}{[\lambda]^{2}[2 \lambda]} \sum_{\kappa(\neq \mu, 0)} \frac{\left[a_{\kappa}+1 / 2+2 \lambda\right]}{\left[a_{\kappa}+1 / 2\right]} G_{a \kappa} .
\end{aligned}
$$

Consider a doubly periodic function

$$
F(z)=\frac{[z+1 / 2+2 \lambda][2 z][z+1][z+3 / 2]}{[z+1 / 2][1][2 z+1][z][z-1 / 2]} \prod_{\kappa(\neq \pm \mu, 0)} \frac{\left[z-a_{\kappa}+1\right]}{\left[z-a_{\kappa}\right]} .
$$

$\sum \operatorname{Res} F(z)=0$ this time gives rise to

$$
\begin{aligned}
& \sum_{\kappa(\neq \mu, 0)} \frac{\left[a_{\kappa}+1 / 2+2 \lambda\right]}{\left[a_{\kappa}+1 / 2\right]} G_{a \kappa}=\frac{1}{2} \sum_{\omega(\neq 0)} \frac{[2 \lambda+\omega]}{[\omega]} e^{2 \pi i \theta(\omega)} \\
& \quad+\frac{[2 \lambda]}{2}\left\{\varphi(2 \lambda)+2 \varphi(1 / 2)+2 \sum_{\kappa(\neq \pm \mu, 0)} \varphi\left(1 / 2-a_{\kappa}\right)\right\} .
\end{aligned}
$$


Furthermore, by specializing $u=-\lambda=-v=-w, a \rightarrow 0$ in Lemma 3 , we have

$$
\sum_{\omega(\neq 0)} \frac{[2 \lambda+\omega]}{[\omega]} e^{2 \pi i \theta(\omega)}=[2 \lambda](2 \varphi(\lambda)-\varphi(2 \lambda)) .
$$

Thanks to (3.7-8) and Lemma 4, (3.6) is reduced to

$$
\frac{[\lambda-1][2 \lambda+1]}{[1][\lambda+1][2 \lambda]}-\frac{[\lambda+1][2 \lambda-1]}{[1][\lambda-1][2 \lambda]}+\frac{[1]^{2}[2 \lambda]}{[\lambda]^{2}[\lambda-1][\lambda+1]}+4 \varphi(\lambda)-2 \varphi(2 \lambda)=0 .
$$

This can be checked by a similar method as in the proof of Lemma 4 .

Now we have proved that $f(1)$ is independent of $a_{\mu}(\mu \neq 0)$. Letting $a_{\mu}=\mu$ for all $\mu$ and using the specialized values

$$
G_{a \kappa}=\frac{[1-2 \lambda]}{[1]} \delta_{\kappa n}, \quad H_{a 0}=\frac{[\lambda+1][2 \lambda-1]}{[1][\lambda-1]},
$$

we find $f(1)=0$. This completes the proof of (iii) with $\mu=\nu=0$.

The remaining case is (iii) with $\mu=0, v \neq 0$. It reads as

$$
\begin{aligned}
0= & \left(\frac{[\lambda+u][2 \lambda-u]}{[\lambda][2 \lambda]}-\frac{[u][\lambda-u]}{[\lambda][2 \lambda]} H_{a 0}\right) \frac{\left[a_{v}+1 / 2+\lambda+u\right]}{\left[a_{v}+1 / 2\right]} \\
& +\sum_{\kappa(\neq v, 0)} \frac{[u][\lambda]}{\left[a_{\kappa}+1 / 2+\lambda-u\right]\left[a_{\kappa}+a_{v}+1+\lambda+u\right]} \\
& -\frac{\left[a_{v}+1 / 2+\lambda-u\right]}{\left[a_{\kappa}+1 / 2\right]\left[a_{\kappa}+a_{v}+1\right]}\left(\frac{[\lambda+u]\left[2 a_{v}+1+u\right]}{[\lambda]\left[2 a_{v}+1\right]}-\frac{[u]\left[2 a_{v}+1+\lambda+u\right]}{[\lambda]\left[2 a_{v}+1\right]} G_{a v}\right) .
\end{aligned}
$$

From Lemma 1, it suffices to check that the right-hand side vanishes at $u=0, \pm \lambda$. The nontrivial case $u=\lambda$ reduces to the identity

$$
\sum_{\kappa} \frac{\left[a_{v}+a_{\kappa}+1+2 \lambda\right]}{\left[a_{v}+a_{\kappa}+1\right]} G_{a \kappa}=\frac{[2 \lambda]\left[2 a_{v}+1+\lambda\right]}{[\lambda]\left[2 a_{v}+1\right]}(v \neq 0) .
$$

This is obtained by specializing $u=\lambda$ in (iii) with $\mu=v \neq 0$.

Remark. From (3.9) it follows also that (2.5e) is equal to (2.5f) in the case $\mu \neq 0$.

Now let us proceed to the proof of the STR. First put

$$
\begin{aligned}
& X(a|\kappa, \mu, v ; \alpha, \beta, \gamma| u, v)
\end{aligned}
$$

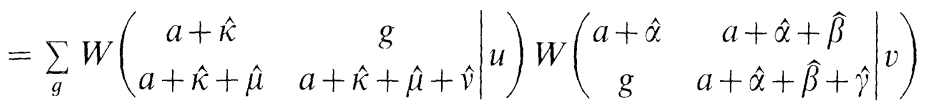

$$
\begin{aligned}
& \times W\left(\begin{array}{cc}
a & a+\hat{\alpha} \\
a+\hat{\kappa} & g
\end{array} \mid u+v\right)-\sum_{g} W\left(\begin{array}{cc}
a & a+\hat{\alpha} \\
g & a+\hat{\alpha}+\hat{\beta}
\end{array} \mid u\right) \\
& \times W\left(\begin{array}{cc}
a & g \\
a+\hat{\kappa} & a+\hat{\kappa}+\hat{\mu}
\end{array} \mid v\right) W\left(\begin{array}{cc}
g & a+\hat{\alpha}+\hat{\beta} \\
a+\hat{\kappa}+\hat{\mu} & a+\hat{\kappa}+\hat{\mu}+\hat{v}
\end{array} \mid u+v\right),
\end{aligned}
$$

where $\hat{\kappa}+\hat{\mu}+\hat{v}=\hat{x}+\hat{\beta}+\hat{\gamma}$ is assumed. 


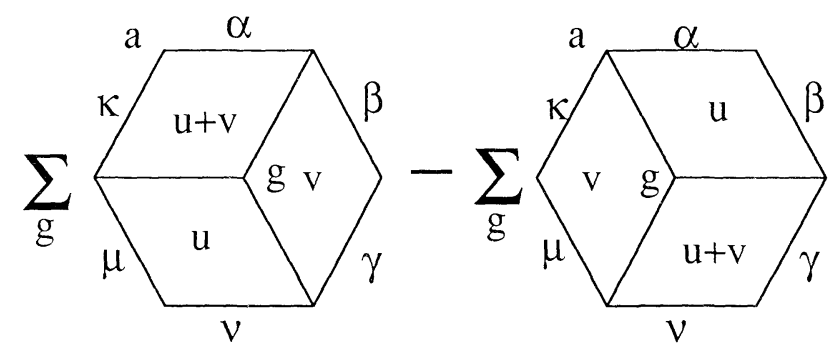

Fig. 1. The type of the STR denoted by $X(a|\kappa, \mu, v ; \alpha, \beta, \gamma| u, v)$

Proposition 5. Set $f(u)=X(a|\kappa, \mu, v ; \alpha, \beta, \gamma| u, v)$. Then $f(u)$ has zeros at $0,-v, \lambda-v$ and $\lambda$.

Proof. That $f(0)=f(-v)=0$ is a direct consequence of the initial condition (2.9) and the inversion relation (2.13a) proved in Proposition 2.

Thanks to the rotational symmetry (2.12), we also have

$X(a|\kappa, \mu, v ; \alpha, \beta, \gamma| u, v)$

$$
=-\left(\frac{G_{a+\hat{\alpha}} G_{a+\hat{\kappa}+\hat{\mu}}}{G_{a} G_{a+\hat{\alpha}+\hat{\beta}+\hat{\gamma}}}\right)^{1 / 2} X(a+\hat{\alpha}|-\alpha, \kappa, \mu ; \beta, \gamma,-v| v, \lambda-u-v) .
$$

This shows $f(\lambda-v)=f(\lambda)=0$.

From (3.1) the Boltzmann weights enjoy the following quasi-periodicity property:

$$
\begin{gathered}
W\left(\begin{array}{ll}
a & b \\
d & c
\end{array} \mid u+L\right)=W\left(\begin{array}{ll}
a & b \\
d & c
\end{array} \mid u\right), \\
W\left(\begin{array}{ll}
a & b \\
d & c
\end{array} \mid u+L \tau\right)=e^{-2 \pi i \tau-2 \pi i(2 u-\lambda+\xi) / L} W\left(\begin{array}{ll}
a & b \\
d & c
\end{array} \mid u\right),
\end{gathered}
$$

where $\xi=1,-a_{\mu}+a_{v}, 0,-a_{\mu}-a_{v}-1,-2 a_{\mu}-1$ for the weights $(2.5 \mathrm{a}-\mathrm{e})$ respectively. From these we have for $f(u)=X(a|\kappa, \mu, v ; \alpha, \beta, \gamma| u, v)$,

$$
\begin{gathered}
f(u+L)=f(u), \\
f(u+L \tau)=e^{-2 \pi i(2 \tau+(4 u+2 v-2 \lambda+\zeta) / L)} f(u),
\end{gathered}
$$

where $\zeta$ depends only on $a_{i}(i=1, \ldots, n)$.

Now let us assume that $\zeta \neq 0 \bmod L \mathbb{Z}+L \mathbb{Z} \tau$. Then we can show that $f(u) \equiv 0$ as follows. We have found zeros at $u=0,-v, \lambda-v$ and $\lambda$. Lemma 1 states that $f(u)$ has exactly four zeros $\bmod L \mathbb{Z}+L \mathbb{Z} \tau$, and that

$$
2 \lambda-2 v \equiv L\left(4-\left(2 \tau+\frac{2 v-2 \lambda+\zeta}{L}\right)\right) \bmod L \mathbb{Z}+L \mathbb{Z} \tau .
$$

This contradicts the assumption $\zeta \neq 0$.

From the symmetry (2.10) and (2.12), the following are equivalent:

$$
\begin{gathered}
X(a|\kappa, \mu, v ; \alpha, \beta, \gamma| u, v)=0, \\
X(a|\alpha, \beta, \gamma ; \kappa, \mu, v| u, v)=0, \\
X(a|\mu, v,-\gamma ;-\kappa, \alpha, \beta| u, v)=0 .
\end{gathered}
$$


Using this, we can reduce the proof of the STR to the case $\zeta \neq 0 \bmod L \mathbb{Z}+L \mathbb{Z} \tau$ except for

$$
\begin{gathered}
X(a|\kappa, \mu, v ; v, \mu, \kappa| u, v)=0 \quad(\kappa \neq \pm \mu, \pm v, \mu \neq \pm v), \\
X(a|0,0,0 ; 0,0,0| u, v)=0, \\
X(a|\mu,-\mu, \mu ; \mu,-\mu, \mu| u, v)=0 \quad(\mu \neq 0), \\
X(a|0, \mu, 0 ; 0, \mu, 0| u, v)=0 \quad(\mu \neq 0) .
\end{gathered}
$$

We now prove these exceptional cases. Equations (1) and (2) follow straightforwardly. Let us consider (3). It has the form $S(a, \mu)-S(a+\hat{\mu},-\mu)=0$, where

$$
\begin{aligned}
S(a, \mu) & =\frac{[u][1+\lambda-u][v][1+\lambda-v][w][1+\lambda-w]}{[\lambda]^{3}[1]^{3}} G_{a \mu}^{-1} G_{a+\hat{\mu}-\mu} \\
& +\left(\frac{[u]\left[2 a_{\mu}+1-\lambda+u\right]}{[\lambda]\left[2 a_{\mu}+1\right]} G_{a+\hat{\mu}-\mu}+\frac{[\lambda-u]\left[2 a_{\mu}+1+u\right]}{[\lambda]\left[2 a_{\mu}+1\right]}\right) \\
& \times\left(\frac{[v]\left[2 a_{\mu}+1-\lambda+v\right]}{[\lambda]\left[2 a_{\mu}+1\right]} G_{a+\hat{\mu}-\mu}+\frac{[\lambda-v]\left[2 a_{\mu}+1+v\right]}{[\lambda]\left[2 a_{\mu}+1\right]}\right) \\
& \times\left(\frac{[\lambda-w]\left[2 a_{\mu}+1+w\right]}{[\lambda]\left[2 a_{\mu}+1\right]} G_{a \mu}+\frac{[w]\left[2 a_{\mu}+1-\lambda+w\right]}{[\lambda]\left[2 a_{\mu}+1\right]}\right) \\
& +\sum_{\kappa(\neq \pm \mu)} \frac{[u]\left[-a_{\mu}+a_{\kappa}+\lambda-u\right][v]\left[-a_{\mu}+a_{\kappa}+\lambda-v\right][w]\left[-a_{\mu}+a_{\kappa}+\lambda-w\right]}{[\lambda]^{3}\left[-a_{\mu}+a_{\kappa}\right]^{3}} \\
& \times G_{a \mu}^{-1} G_{a+\hat{\mu}-\kappa} .
\end{aligned}
$$

Here $w=\lambda-u-v$.

Now consider a function

$$
\begin{aligned}
F(z)= & \frac{C\left[-a_{\mu}+z+\lambda-u\right]\left[-a_{\mu}+z+\lambda-v\right]\left[-a_{\mu}+z+\lambda-w\right]}{\left[-a_{\mu}+z\right]^{3}} \\
& \times \frac{\sigma h(z+1)[2 z]}{[1] h(z)[2 z+1]} \prod_{\kappa(\neq \pm \mu, 0)} \frac{\left[z-a_{\kappa}+1\right]}{\left[z-a_{\kappa}\right]} .
\end{aligned}
$$

This is doubly periodic. A similar calculation as before shows $\left(A=2 a_{\mu}+1\right)$

$$
\begin{aligned}
& (S(a, \mu)-S(a+\hat{\mu},-\mu)) \frac{[\lambda]^{3}}{3} \\
& \quad=-\frac{[2 A]}{[A]^{4}}+\frac{[A-1][A+1]}{[1]^{2}[A]^{2}}(\varphi(A+1)+\varphi(A-1)-2 \varphi(A)) .
\end{aligned}
$$

This vanishes by virtue of Lemma 4 .

The proof of (4) reduces to the identity

$$
H_{a 0}-H_{a+\hat{\mu} 0}=\frac{[1]^{2}[2 \lambda]\left[2 a_{\mu}+1\right]}{\left[a_{\mu}-1 / 2\right]\left[a_{\mu}+1 / 2\right]^{2}\left[a_{\mu}+3 / 2\right]},
$$

where $H_{a 0}$ is given in $(2.5 \mathrm{~g})$. Without loss of generality we can assume that $\mu=n$. The function $H_{a 0}$ is doubly periodic in each variable $a_{1}, \ldots, a_{n}$. The poles in $a_{i}$ 
within the period rectangle are $a_{i}= \pm 1 / 2$ and their residues are independent of $\left(a_{1}, \ldots, a_{n}\right)$. Therefore $H_{a 0}-H_{a+\hat{n} 0}$ is independent of $\left(a_{1}, \ldots, a_{n-1}\right)$. By setting $a_{i}$ $=i(i=1, \ldots, n-1),(3.10)$, reduces to $\left(A=a_{n}\right)$,

$$
\begin{aligned}
& \frac{[1]^{2}[1-2 n][1+2 A]}{[A-1 / 2][A+1 / 2]^{2}[A+3 / 2]} \\
& =\frac{[2 n-2]}{[1]}\left(\frac{[n+1+A][n-1-A]}{[n+A][n-2-A]}-\frac{[n+A][n-A]}{[n-1+A][n-1-A]}\right) \\
& \quad+\sum_{B=A, A+1}(-)^{B-A}\left(-\frac{[2 n-2][n+B][n-B]}{[1][n-1+B][n-1-B]}\right. \\
& \left.\quad+\frac{[B+3 / 2-2 n][B+n]}{[B+1 / 2][B-n+1]}+\frac{[B-3 / 2+2 n][B-n]}{[B-1 / 2][B+n-1]}\right) .
\end{aligned}
$$

It is easy to see that (the left-hand side)-(the right-hand side) is independent of $A$. Setting $A=n$ we can check the difference is vanishing.

Thus we have completed the proof of the STR.

\section{Restricted Models}

Let $X_{n}$ denote $A_{n}(n \geqq 1), B_{n}(n \geqq 2), C_{n}(n \geqq 1)$ or $D_{n}(n \geqq 3)$. In this section we construct solvable face models whose local states are the dominant integral weights of the affine Lie algebra $X_{n}^{(1)}$ of a fixed level $l$. Our procedure is to restrict the models discussed in the previous sections as follows. We set

$$
L=t(l+g),
$$

where $t$ is given in Table 2. By definition a local state $a$ of level $l$ is a level $l$ dominant integral weight of $X_{n}^{(1)}$. It reads as follows:

$$
A_{n}^{(1)}: \quad a=\left(L-a_{1}+a_{n+1}-1\right) \Lambda_{0}+\sum_{i=1}^{n}\left(a_{i}-a_{i+1}-1\right) \Lambda_{i},
$$

where $a_{i}-a_{j} \in \mathbb{Z}, \sum_{i=1}^{n+1} a_{i}=0$, and $L+a_{n+1}>a_{1}>a_{2}>\ldots>a_{n+1}$.

$B_{n}^{(1)}: \quad a=\left(L-a_{1}-a_{2}-1\right) \Lambda_{0}+\sum_{i=1}^{n-1}\left(a_{i}-a_{i+1}-1\right) \Lambda_{i}+\left(2 a_{n}-1\right) \Lambda_{n}$,

where either $a_{i} \in \mathbb{Z}$ (all $i$ ) or $a_{i} \in \mathbb{Z}+1 / 2$ (all $i$ ), and $L>a_{1}+a_{2}, a_{1}>a_{2}>\ldots>a_{n}>0$.

$C_{n}^{(1)}: \quad a=\left(L / 2-a_{1}-1\right) \Lambda_{0}+\sum_{i=1}^{n-1}\left(a_{i}-a_{i+1}-1\right) \Lambda_{i}+\left(a_{n}-1\right) \Lambda_{n}$,

where $a_{i} \in \mathbb{Z}$ and $L / 2>a_{1}>a_{2}>\ldots>a_{n}>0$.

$D_{n}^{(1)}: \quad a=\left(L-a_{1}-a_{2}-1\right) \Lambda_{0}+\sum_{i=1}^{n-1}\left(a_{i}-a_{i+1}-1\right) \Lambda_{i}+\left(a_{n-1}+a_{n}-1\right) \Lambda_{n}$,

where either $a_{i} \in \mathbb{Z}($ all $i)$ or $a_{i} \in \mathbb{Z}+1 / 2$ (all $\left.i\right)$, and $L>a_{1}+a_{2}, a_{1}>a_{2}>\ldots>a_{n}, a_{n-1}$ $+a_{n}>0$. 
Let $\bar{a}$ denote the classical part of $a$, and let $V(\bar{a})$ be the irreducible $X_{n}$ module with the highest weight $\bar{a}$. A pair of weights $(a, b)$ is called admissible if and only if $a, b$ are local states of level $l$ and $V(\sigma(\bar{b}))$ appears in the tensor module $V(\sigma(\bar{a})) \otimes V\left(\bar{\Lambda}_{1}\right)$ for any Dynkin diagram automorphism. (The representation $V\left(\bar{\Lambda}_{1}\right)$ is called the vector representation of $X_{n}$.) This condition is equivalent to $b-a \in \mathscr{A}$ (see Table 1) except for the case $X_{n}=B_{n}$ with $a=b$ and $a_{n}=1 / 2$; in this case $(a, b)$ is non-admissible because $V(\bar{a})$ is not contained in $V(\bar{a}) \otimes V\left(\bar{\Lambda}_{1}\right)$. Note also that if $(a, b)$ is admissible the multiplicity of $V(\bar{b})$ in $V(\bar{a}) \otimes V\left(\bar{\Lambda}_{1}\right)$ is exactly one.

We define the restricted weight of a configuration $\left(\begin{array}{ll}a & b \\ d & c\end{array}\right)$ round a face to be $W\left(\begin{array}{ll}a & b \\ d & c\end{array} \mid u\right)$ as given in $(2.4)$ and $(2.5)$ if $(a, b),(b, c),(a, d),(d, c)$ are admissible, and to be 0 otherwise.

Theorem 7. The restricted weights are finite and satisfy the STR among themselves.

Proof. We consider the case $X_{n}^{(1)}=B_{n}^{(1)}, C_{n}^{(1)}, D_{n}^{(1)}$. The $A_{n}^{(1)}$ case is similar. First we show that $W\left(\begin{array}{ll}a & b \\ d & c\end{array} \mid u\right)$ in (2.5) is finite if $a$ is a local state and not both $(a, b)$ and $(a, d)$ are non-admissible. The factor $G_{a \mu}$ of (2.7) is finite because $0<\left|a_{\mu}+a_{v}\right|<L$ if $\mu \neq \pm v$ and $\mu, v \neq 0$. (Note also that $0<\left|2 a_{\mu}\right|<L$ if $X_{n}=C_{n}$.)

The weight of type $\mu \square_{v}^{\mu} v$ or $\mu \square_{v}^{v} \mu$ with $\mu \neq \pm v$ is finite if $\left[a_{\mu}-a_{v}\right] \neq 0$. So it is except for the case $a_{\mu}=1 / 2$ and $(\mu, v)=(0,-n),(-n, 0)$, in which both $(a, b)$ and $(a, d)$ are non-admissible. The weight of type $0 \square 0$ is finite because $\left[a_{\mu}+1 / 2\right]=0$ means that $\mu=-n, a_{n}=1 / 2$ and that both $(a, b)$ and $(a, d)$ are non-admissible. The weight of type $\mu \square_{-\mu}^{v}-v(\mu \neq v)$ is finite because the factor $\left[a_{\mu}+a_{v}+1\right]$ is cancelled by the same factor in $\sqrt{G_{a \mu} G_{a v}}$. Finally the weight of type $\mu \square_{-\mu}^{\mu}-\mu(\mu \neq 0)$ is finite because we have two different expressions (2.5e) and (2.5f); the former is relevant if $\left[2 a_{\mu}+1+2 \lambda\right] \neq 0$ and so is the latter if $\left[2 a_{\mu}+1\right] \neq 0$. (Note that $\left[a_{\mu}+a_{\kappa}+1\right]$ $(\kappa \neq \pm \mu)$ in $(2.5 \mathrm{~g})$ is cancelled by $\left.G_{a \kappa}.\right)$

Next we prove that if $(a, d),(d, c)$ are admissible and $(a, b)$ is non-admissible then the weight $W\left(\begin{array}{ll}a & b \\ d & c\end{array} \mid u\right)$ is vanishing with the exceptions mentioned below. As for the weight of type $\mu \square \mu(\mu \neq \pm v)$ this is because $\left[a_{\mu}-a_{v}-1\right]=0$. As for the weight of type $\mu \underset{-\mu}{v}-v(\mu \neq v)$ this is because $G_{a v}=0$ unless $X_{n}=B_{n}, a_{n}=1 / 2$ and $v=0,-\mathrm{n}$. Since the original weights $W\left(\begin{array}{ll}a & b \\ d & c\end{array} \mid u\right)$ satisfy the STR, the proof of the theorem is completed if it is shown that the contribution from the terms with non- 


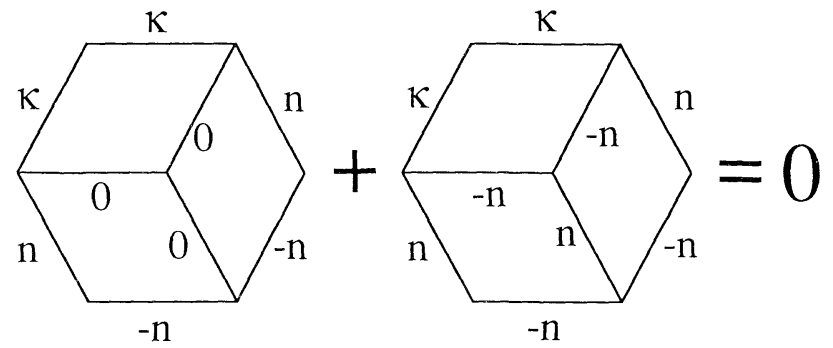

Fig. 2. The cancellation of the two unwanted terms in the STR

admissible pairs is zero. With the above consideration the last step is to check the cancellation of Fig. 2 in the case $X_{n}=B_{n}$ and $b_{n}=f_{n}=1 / 2$. This is straightforward.

\section{Discussion}

In this paper we have presented further elliptic solutions to the STR. They give solvable models with face interactions on a two dimensional square lattice $\mathscr{L}$. The local states of the model belong to the dual space of the Cartan subalgebra of the affine Lie algebra $X_{n}^{(1)}=A_{n}^{(1)}, B_{n}^{(1)}, C_{n}^{(1)}$ or $D_{n}^{(1)}$. Two local states $a, b$ are admitted to occupy adjacent sites of $\mathscr{L}, a$ being located at the left or the upper neighbor of $b$, if and only if $b-a$ is a weight of the vector representation of $X_{n}$. We propose to call the model the $X_{n}^{(1)}$ face model (or the unrestricted face model of type $X_{n}^{(1)}$ ).

The naming comes from the fact that a hierarchy of restricted models is obtained from the unrestricted one in such a way that the local states are the level $l$ dominant integral weights of $X_{n}^{(1)}$.

We note that a solvable face model is not uniquely determined by the specification of the local states and the selection rule of adjacent states. In fact, the unrestricted models of type $C_{n}^{(1)}$ and $D_{n}^{(1)}$ are not distinguishable in this sense. There are three different restricted models with the same local states and the selection rule of Fig. 3; the Akutsu-Kuniba-Wadati model [24], the level 2 restricted model of type $B_{n}^{(1)}$ with $a_{i} \in \mathbb{Z}+1 / 2$ and the level 2 restricted model of type $D_{2 n+1}^{(1)}$ with $a_{i} \in \mathbb{Z}$. From our unrestricted models the whole series of restricted ones are produced simultaneously by restricting the local states $a$ and the parameter $L$. We think this fact justifies our naming.

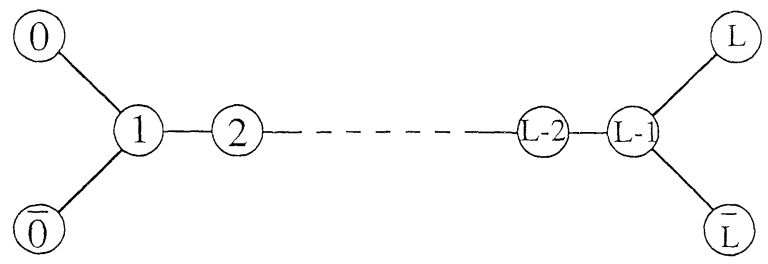

Fig. 3. The incidence diagram common to three models, the Akutsu-Kuniba-Wadati model, the $B_{n}^{(1)}$ face model and the $D_{2 n+1}^{(1)}$ face model $(L=2 n+1)$ 
It will be fully supported if the following programs can be executed:

(i) The construction of unrestricted models of type $X_{n}^{(1)}$ (including $E_{6}^{(1)}, E_{7}^{(1)}$, $E_{8}^{(1)}, G_{2}^{(1)}$, and $\left.F_{4}^{(1)}\right)$ corresponding to arbitrary irreducible representations of $X_{n}$, and the restriction to level $l$. In general, the weight multiplicities of an irreducible representation of $X_{n}$ may exceed one. For such a case a modification of the STR will be necessary.

(ii) The computation of the local state probabilities: The symmetric tensor of the $A_{1}^{(1)}$ face model $[17,18]$ and the vector representation of the $A_{n}^{(1)}$ face model [20] have been treated. The results were mysteriously related to the irreducible decomposition of the tensor product representations of $A_{n}^{(1)}$. A key to this link was the identification of the factors $G_{a}(2.8)$ in the second inversion relation with the specialized characters of $A_{n}^{(1)}$. It is tantalizing to note that the $G_{a}$ of the $X_{n}^{(1)}$ face models coincide with the specialized characters of the dual affine Lie algebra of $X_{n}^{(1)}$, i.e. $A_{2 n-1}^{(2)}$ for $B_{n}^{(1)}$ and $D_{n+1}^{(2)}$ for $C_{n}^{(1)}$. ( $D_{n}^{(1)}$ is self dual.) We hope to discuss on this point in a future publication.

Before ending, a remark is in order about the relation with solvable vertex models. In $[19,20]$ we have found the $A_{n}^{(1)}$ face models via the vertex-face correspondence [38] starting from Belavin's elliptic solution [39]. Is there a similar correspondence in the $B_{n}^{(1)}, C_{n}^{(1)}, D_{n}^{(1)}$ cases? In [5] a classification scheme was given of the solutions to the classical Yang-Baxter equation in terms of simple Lie algebras. Belavin's solution mentioned above corresponds to the elliptic solution of type $A_{n}$ in their table. Under the non-degeneracy condition assumed by Belavin-Drinfeld no other elliptic solutions exist. Therefore, if possibly the vertexface correspondence can be extended to the general cases the vertex counterpart must not have the classical limit in Belavin-Drinfeld's sense. We also note that the trigonometric limit of the vertex-face correspondence, between Belavin's vertex model and the $A_{n}^{(1)}$ face model, becomes trivial and does not give a proper correspondence between the models in the limit. For the moment we do not know any internal link between the trigonometric vertex models of [39-41] and the trigonometric limit of the face models given in this paper.

Acknowledgements. We would like to thank A. Tsuchiya, Y. Kanie, A. Kuniba, and T. Yajima for useful discussions.

\section{References}

1. Kuniba, A., Akutsu, Y., Wadati, M.: Virasoro algebra, von Neumann algebra and critical eight-vertex SOS models. J. Phys. Soc. Jpn. 55 3285-3288 (1986)

2. Akutsu, Y., Wadati, M.: Knot invariants and critical statistical systems, J. Phys. Soc. Jpn. 56 839-842 (1987)

3. Kohno, T.: Monodromy representations of braid groups and Yang-Baxter equations, preprint 1987. Ann. Inst. Fourier (to appear)

4. Turaev, V.G.: The Yang-Baxter equation and invariants of links, LOMI preprint E-3-87, 1987

5. Belavin, A.A., Drinfeld, V.G.: Solutions of the classical Yang-Baxter equation for simple Lie algebras. Funct. Anal. Appl. 16 159-180 (1982)

6. Kulish, P.P., Reshetikhin, N.Yu., Sklyanin, E.K.: Yang-Baxter equation and representation theory. I. Lett. Math. Phys. 5, 393 (1981)

7. Sklyanin, E.K.: Some algebraic structure connected with the Yang-Baxter equation. Funct. Anal. Appl. 16, 263-270 (1982); 17, 273-284 (1983) 
8. Kulish, P.P., Reshetikhin, N.Yu.: Quantum linear problem for the sine-Gordon equation and higher representations. J. Sov. Math. 23, 2435-2441 (1983)

9. Drinfeld, V.G.: Quantum groups, ICM 86 report

10. Jimbo, M.: A $q$-difference analogue of $U(\mathfrak{g})$ and the Yang-Baxter equation. Lett. Math. Phys. 10, 63-69 (1985)

11. Jimbo, M.: A $q$-analogue of $U(g I(N+1))$, Hecke algebra, and the Yang-Baxter equation. Lett. Math. Phys. 11, 247-252 (1986)

12. Baxter, R.J.: Exactly solved models in statistical mechanics. London: Academic 1982

13. Baxter, R.J.: Hard hexagons: Exact solution. J. Phys. A: Math. Gen. 13, L 61-L 70 (1980)

14. Andrews, G.E., Baxter, R.J., Forrester, P.J.: Eight-vertex SOS model and generalized RogersRamanujan-type identities. J. Stat. Phys. 35, 193-266 (1984)

15. Date, E., Jimbo, M., Miwa, T., Okado, M.: Automorphic properties of local height probabilities for integrable solid-on-solid models. Phys. Rev. B 35, 2105-2107 (1987)

16. Jimbo, M., Miwa, T., Okado, M.: Solvable lattice models with broken $\mathbb{Z}_{n}$ symmetry and Hecke's indefinite modular forms. Nucl. Phys. B 275 [FS 17], 517-545 (1986)

17. Date, E., Jimbo, M., Kuniba, A., Miwa, T., Okado, M.: Exactly solvable SOS models: Local height probabilities and theta function identies, Nucl. Phys. B 290 [FS 20], 231-273 (1987)

18. Date, E., Jimbo, M., Kuniba, A., Miwa, T., Okado, M.: Exactly solvable SOS models. II. Proof of the star-triangle relation and combinatorial identities. Adv. Stud. Pure Math. 16 (to appear)

19. Jimbo, M., Miwa, T., Okado, M.: Solvable lattice models whose states are dominant integral weights of $A_{n-1}^{(1)}$, Lett. Math. Phys. 14, 123-131 (1987)

20. Jimbo, M., Miwa, T., Okado, M.: Local state probabilities of solvable lattice models: An $A_{n-1}^{(1)}$ family, preprint RIMS-594, Kyoto Univ. (1987). Nucl. Phys. (to appear)

21. Baxter, R.J., Andrews, G.E.: Lattice gas generalization of the hard hexagon model. I. Startriangle relation and the local densities. J. Stat. Phys. 44, 249-271 (1986)

22. Kuniba, A., Akutsu, Y., Wadati, M.: Exactly solvable IRF models. I. A three state model, J. Phys. Soc. Jpn. 55, 1092-1101 (1986)

23. Akutsu, Y., Kuniba, A., Wadat1, M.: Exactly solvable IRF models. II. $S_{N}$ generalizations. J. Phys. Soc. Jpn. 55, 1466-1474 (1986)

24. Akutsu, Y., Kuniba, A., Wadati, M.: Exactly solvable IRF models. III. A new hierarchy of solvable models. J. Phys. Soc. Jpn. 55, 1880-1886 (1986)

25. Date, E., Jimbo, M., Miwa, T., Okado, M.: Fusion of the eight-vertex SOS model, Lett. Math. Phys. 12, 209-215 (1986)

26. Jimbo, M., Miwa, T., Okado, M.: An $A_{n-1}^{(1)}$ family of solvable lattice models. Mod. Phys. Lett. B 1, 73-79 (1987)

27. Jimbo, M., Miwa, T., Okado, M.: Symmetric tensors of the $A_{n-1}^{(1)}$ family, preprint RIMS 592, Kyoto University (1987). Algebraic Analysis (Festschrift for M. Sato's 60th birthday) Academic Press 1988

28. Pasquier, V.: Exact solubility of the $D_{n}$ series. J. Phys. A: Math. Gen. 20, L217-L220 (1987)

29. Kashiwara, M., Miwa, T.: A class of elliptic solutions to the star-triangle relation. Nucl. Phys. B 275 [FS17], 121-134 (1986)

30. Kuniba, A., Yajima, T.: Local state probabilities for solvable RSOS models: $A_{n}, D_{n}, D_{n}^{(1)}$, and $A_{n}^{(1)}$, preprint. Tokyo University 1987

31. Wenzl, H.: Representations of Hecke algebras and subfactors. Thesis, University of Pennsylvania 1985

32. Tsuchiya, A., Kanie, Y.: Vertex operators on conformal field theory on $\mathbb{P}^{1}$ and monodromy representations of braid groups. Adv. Stud. Pure Math. 16 (to appear)

33. Pasquier, V.: Continuum limit of lattice models built on quantum groups. Saclay preprint SPHT/87-125

34. Kac, V.G., Peterson, D.H.: Infinite-dimensional Lie algebras, theta functions and modular forms. Adv. Math. 53, 125-264 (1984)

35. Bourbaki, N.: Groupes et Algebres de Lie, Chaps. 4-6. Paris: Hermann 1968

36. Cherednik, I.V.: Some finite dimensional representations of generalized Sklyanin algebra. Funct. Anal. Appl. 19, 77-79 (1985) 
37. Richey, M.P., Tracy, C.A.: $\mathbb{Z}_{n}$ Baxter model: symmetries and the Belavin parametrization. J. Stat. Phys. 42, 311-348 (1986)

38. Baxter, R.J.: Eight-vertex model in lattice statistics and one-dimensional anisotropic Heisenberg chain. II. Equivalence to a generalized ice-type lattice model, ibid. 76, 25-47 (1973)

39. Belavin, A.A.: Dynamical symmetry of integrable quantum systems. Nucl. Phys. B 180 [FS 2], 189-200 (1981)

40. Bazhanov, V.V.: Trigonometric solutions of the triangle equation and classical Lie algebras. Phys. Lett. 159 B, 321-324 (1985)

41. Jimbo, M.: Quantum $R$ matrix for the generalized Toda system. Commun. Math. Phys. 102, $537-547(1986)$

42. Jimbo, M.: Quantum $R$ matrix related to the generalized Toda system: an algebraic approach. In: Proceedings of the Symposium on field theory, quantum gravity and strings. Meudon and Paris VI, 1984/85. Lecture Notes in Physics, Vol. 246, pp. 335-361. Berlin, Heidelberg, New York: Springer 1986

Communicated by H. Araki

Received December 8, 1987 
